\title{
CROSS WORD PUZZLE
}

Surantha Perera ${ }^{1}$,Probhodana Ranaweera ${ }^{2}$

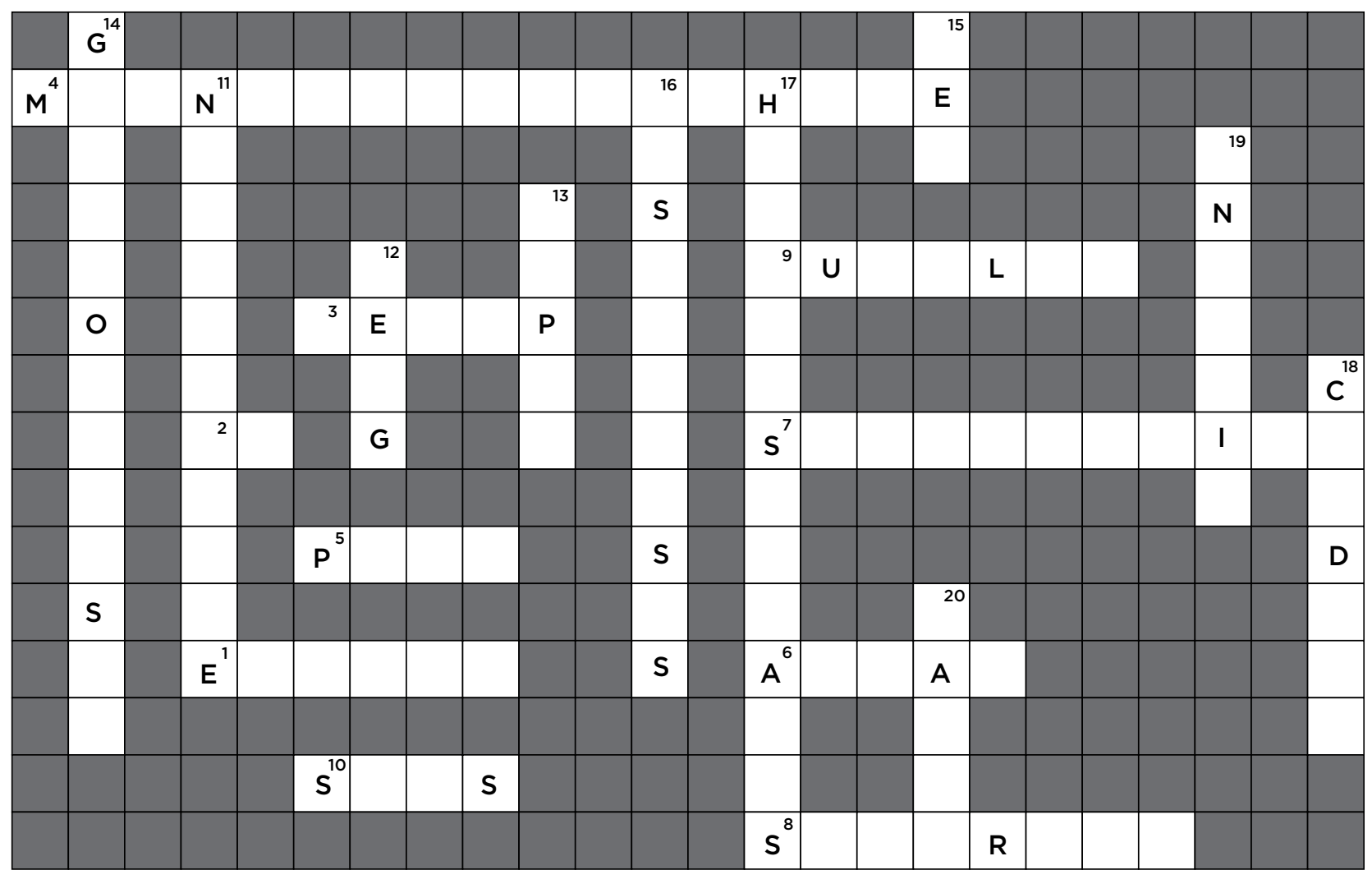

Across

1. Trisomy 18

2. Calculated to determine ECMO

3. A condition associated with Pre-eclampsia

4. A medication used in preterm labour and eclampsia

5. Occurs due to ascending infection in pregnancy

6. It is an assessment done at the birth

7. Lack of folic acid causeS this

8. A group of clinical symptoms which occur consistently

9. Intrauterine infection causing heart disease

10. provides better view of the woman cervix

\section{${ }^{I}$ Consultant Paediatrician}

Castle Street Hospital for Women, Colombo,

Sri Lanka

${ }^{2}$ Senior Lecturer Department of Obstetrics

\& Gynaecology, University of Colombo,

Sri Lanka

\section{Down}

11. Potent pulmonary vasodilator

12. Gold standard for monitoring neonatal convulsions

13. Maternal illness causes heart block in newborn

14. A Birth defect in which babies' intestines extend outside of the abdomen

15. In this condition gas is seen in the intestinal wall

16. preterm meconium stained liquor seen in this condition

17. Associated with Parvo virus B19

18. Commonest vaginal infection during pregnancy

19. Occurs commonly due to Iron deficiency in pregnancy

20. Heart shadow of figure of eight seen in CXR of a newborn 